\title{
Aspectos psicológicos e qualidade de vida em pacientes com melanoma uveal durante o processo de tratamento por remoção do bulbo ocular
}

\author{
Psychological aspects and quality oflife in uveal melanoma patients during the \\ treatment process by ocularbulb remotion
}

\author{
Terezinha Aparecida Carvalho Amaro ${ }^{1}$ \\ Latife Yazigi' ${ }^{2}$ \\ Clélia Erwenne ${ }^{3}$
}

Doutorando do Setor de Oncologia Ocular do Departamento de Oftalmologia da Universidade Federal de São Paulo - UNIFESP - São Paulo (SP) - Brasil.

Professor Titular do Departamento de Psiquiatria da UNIFESP - São Paulo (SP) - Brasil.

Professor Doutor do Setor de Oncologia Ocular do Departamento de Oftalmologia da UNIFESP - São Paulo (SP) - Brasil.

Endereço para correspondência: Terezinha Aparecida Carvalho Amaro. Rua França Pinto, 756/54 - São Paulo (SP) CEP 04016-003

E-mail: t_amaro@terra.com.br

Recebido para publicação em 18.08.2005

Última versão recebida em 03.05 .2006

Aprovação em 18.05.2006

Nota Editorial: Depois de concluída a análise do artigo sob sigilo editorial e com a anuência do Dr. Eduardo Ferrari Marback sobre a divulgação de seu nome como revisor, agradecemos sua participação neste processo.

\section{RESUMO}

Objetivo: Avaliar prospectivamente as repercussões emocionais no indivíduo com melanoma uveal e indicação cirúrgica em três momentos: diagnóstico e pré-cirúrgico, pós-cirúrgico e pós-cirúrgico tardio. Métodos: Trata-se de estudo clínico-qualitativo, no qual se utilizaram os seguintes instrumentos: Inventário de Ansiedade Traço/Estado - IDATE; Inventário de Depressão Beck e o Questionário de Qualidade de Vida SF-36. Resultados: Participaram do estudo, inicialmente, 20 pacientes, 13 homens e sete mulheres, com idade média de 52 anos e, num segundo momento, 16 estavam disponíveis. No momento pré-cirúrgico, o Estado de Ansiedade está aumentado em relação ao Traço. O índice de Depressão indica um estado mínimo a leve e a Qualidade de Vida revela que os aspectos físicos e emocionais são os mais afetados. No pós-cirúrgico de três meses, o Traço de Ansiedade está consideravelmente elevado, o índice de Depressão mostra um estado leve a grave e na Qualidade de Vida os aspectos mais atingidos são os físicos, sociais e emocionais, além da vitalidade e da saúde mental. No pós-cirúrgico de um ano, o Traço de Ansiedade se mantém semelhante ao da primeira avaliação, o Estado de Ansiedade diminui consideravelmente, a Depressão cai para umíndice mínimo e a Qualidade de vida mostra a maioria dos aspectos equilibrados. Conclusões: Após três meses da cirurgia, os pacientes se mostram mais frágeis, com dificuldade de adaptação, com aumento do grau de depressão e ansiedade. Esse foi o pior período para os pacientes. Após um ano da cirurgia, mostram-se mais estruturados e com os aspectos de Qualidade de Vida mais equilibrados.

Descritores: Neoplasias uveais/complicações; Neoplasias uveais/psicologia; Enucleação ocular/etiologia; Enucleação ocular/psicologia; Ansiedade/psicologia; Depressão/psicologia; Qualidade de vida

\section{INTRODUÇÃOO}

Apesar de uma consciência crescente, poucos são os estudos sistemáticos voltados para a orientação do clínico em face do impacto do câncer sobre o paciente e sobre a violência dos processos cirúrgicos decorrentes. Em oncologia, a cirurgia radical é muitas vezes necessária, mas pode levar a severos prejuízos funcionais e eventualmente estéticos ${ }^{(1)}$.

O presente estudo se propõe a avaliar prospectivamente pacientes, com melanoma uveal e indicação para enucleação, nos aspectos psicológicos em três momentos: diagnóstico e indicação cirúrgica, pós-cirúrgico e póscirúrgico tardio. 


\section{MÉTODOS}

Pacientes deste estudo foram atendidos no período de 2001 a 2003, no Setor de Oncologia do Departamento de Oftalmologia da Universidade Federal de São Paulo (UNIFESP).

Critérios de inclusão: diagnóstico de melanoma uveal em um dos olhos, e:

1) Indicação de enucleação desse olho.

2) Visão no olho contralateral suficiente para desempenho autônomo das funções cotidianas (acuidade visual corrigida de 20/40, ou melhor).

3) Ausência de metástases sistêmicas.

4) Possibilidade de cirurgia com especialista em oftalmologia e plástica ocular com colocação de implante orbitário poroso e prótese externa com bom prognóstico quanto ao resultado estético pós-operatório.

Instrumentos da avaliação psicológica: a avaliação se baseia no método clínico qualitativo(2).

1) Inventário de Ansiedade Traço-Estado/ IDATE ${ }^{(3)}$ STAI - State Trait Anxiety Inventory

2) Inventário de Depressão Beck / BDI - Beck Depression Inventory ${ }^{(4)}$

3) Questionário de Qualidade de vida SF-36/ Medical Outcomes Study 36 - Item Short-Form Health Survey ${ }^{(5)}$

As avaliações psicológicas foram feitas pelo psicólogo pesquisador deste estudo que, inicialmente, aplicou os instrumentos na ordem descrita acima, ou seja, por ocasião do diagnóstico e indicação cirúrgica, aos três e aos 12 meses do pós-operatório. A avaliação do Traço de Ansiedade foi realizada apenas nos momentos pré-cirúrgico e pós-operatório tardio.

A cirurgia foi realizada sempre pelo mesmo cirurgião; os implantes porosos foram sempre os mesmos (Porex ${ }^{\circledR}$ ). O protocolo cirúrgico foi padronizado para se obter bom preenchimento da cavidade orbitária e movimentação da esfera porosa. Essa mesma amostra de pacientes foi objeto de estudo da tese de doutorado do cirurgião na área óculo-plástica. Os pacientes foram previamente informados dos passos do procedimento cirúrgico e da expectativa de boa recuperação estética.

\section{RESULTADOS}

Foram avaliados individualmente 20 pacientes. A idade média foi de 52 anos, com variação dos 29 aos 80 anos, 13 eram do sexo masculino (65\%); deles, 15 (75\%) eram casados, dois solteiros, dois divorciados e um viúvo. Quanto à escolaridade, $60 \%$ não haviam completado o ensino fundamental, $25 \%$ tinham concluído o ensino médio e $15 \%$ o nível superior.

Participaram da primeira e segunda avaliação os 20 pacientes, porém na terceira tomaram parte 16 pacientes, uma vez que um paciente faleceu, outro teve um acidente vascular cerebral (AVC) e dois mudaram para outras cidades e abandonaram o atendimento.

\section{1) Inventário de Ansiedade Traço - Estado/ IDATE}

O IDATE consta de 20 itens separados para medir o Traço e o Estado de Ansiedade e a contagem individual varia de 1 a 4. A contagem total para cada escala varia de 20 a 80 e as contagens mais elevadas denotam altos níveis de Ansiedade. O ponto de interrupção para classificar a Ansiedade elevada ou baixa é o quartile. Nesta avaliação, como nível de corte, utilizamos o estudo feito com adultos em período pré-operatório ${ }^{(6)}$ que considerou a média para o Traço de Ansiedade a pontuação 38 e para o Estado de Ansiedade a pontuação 39.

O Traço de Ansiedade avaliado no momento do diagnóstico foi superior à média esperada $(>38)$ em 13 pacientes $(65 \%)$. Foi estimado na média em $2(10 \%)$ e ficou abaixo da média em 5 (25\%). O valor médio calculado para o grupo de 20 pacientes foi de 40,6. Na terceira avaliação (pós-cirúrgico tardio) reavaliou-se o Traço de Ansiedade, desta feita em apenas 16 pacientes. Observou-se que ele estava acima da média em 6 (37,5\%); abaixo da média em 8 (50\%) e na média em 2 pacientes $(12,5 \%)$. O valor médio calculado para o grupo foi de 39 (Quadro 1).

O Estado de Ansiedade, na primeira avaliação, era elevado em relação à média esperada $(>39)$ em 17 pacientes $(85 \%)$. O valor médio do grupo de 20 pacientes foi de 47,6. Na segunda avaliação, o Estado de Ansiedade aumentou em 12 pacientes (60\%) levando o valor médio para 51,9. Na terceira avaliação, 14 dos 16 pacientes em que esta foi possível (87,5\%) evoluíram com melhora do Estado de Ansiedade, o que se refletiu na queda do valor médio do grupo, para 41 (Quadro 1).

\begin{tabular}{|c|c|c|c|c|c|}
\hline \multicolumn{6}{|c|}{$\begin{array}{l}\text { Quadro 1. IDATE - Inventário de Ansiedade Traço/Estado. Avaliação } \\
\text { da Ansiedade nos momentos: pré e pós-cirúrgico }\end{array}$} \\
\hline \multirow[t]{2}{*}{ Pacientes } & \multicolumn{2}{|c|}{ Pré-cirúrgico } & \multirow{2}{*}{$\begin{array}{c}\text { Pós-cirúrgico } \\
3 \text { meses } \\
\text { Estado }\end{array}$} & \multicolumn{2}{|c|}{$\begin{array}{l}\text { Pós-cirúrgico } \\
1 \text { ano }\end{array}$} \\
\hline & Traço & Estado & & Traço & Estado \\
\hline 1 & 45 & 48 & 46 & * & * \\
\hline 2 & 46 & 49 & 61 & 38 & 40 \\
\hline 3 & 38 & 53 & 56 & 38 & 59 \\
\hline 4 & 50 & 53 & 55 & 42 & 44 \\
\hline 5 & 36 & 45 & 56 & 40 & 41 \\
\hline 6 & 42 & 55 & 67 & 43 & 35 \\
\hline 7 & 39 & 37 & 49 & $* *$ & $* *$ \\
\hline 8 & 39 & 43 & 43 & 37 & 33 \\
\hline 9 & 38 & 40 & 37 & 37 & 31 \\
\hline 10 & 31 & 46 & 66 & 40 & 42 \\
\hline 11 & 44 & 50 & 37 & $* * *$ & *** \\
\hline 12 & 44 & 48 & 36 & 39 & 33 \\
\hline 13 & 44 & 53 & 45 & 39 & 42 \\
\hline 14 & 44 & 60 & 64 & 46 & 50 \\
\hline 15 & 39 & 66 & 66 & 41 & 42 \\
\hline 16 & 37 & 37 & 41 & 32 & 32 \\
\hline 17 & 32 & 32 & 40 & 37 & 43 \\
\hline 18 & 46 & 51 & 53 & 35 & 42 \\
\hline 19 & 43 & 49 & 75 & 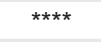 & $\star * \star \star *$ \\
\hline 20 & 34 & 42 & 42 & 34 & 42 \\
\hline
\end{tabular}




\section{2) Inventário de Depressão Beck}

As avaliações de Depressão por meio do BDI, Inventário de Depressão Beck, identificam o estado mínimo de Depressão: pontuação de 0 a 11; Depressão leve: pontuação de 12 a 19; Depressão moderada: pontuação de 20 a 35; e Depressão grave: pontuação de 36 a 63 .

No primeiro momento deste estudo, $11(55 \%)$ dos 20 pacientes apresentaram estado de Depressão mínimo; 7 (35\%) Depressão leve, e 2 (10\%) mostraram Depressão moderada. Na segunda avaliação: o estado de Depressão mínimo se manteve em 4 pacientes (20\%); o de Depressão leve em 9 (45\%); o de Depressão moderada em 5 (25\%) e apenas $2(10 \%)$ com Depressão grave.

$\mathrm{Na}$ terceira avaliação, com 16 pacientes, (14 ou 87,5\%) apresentaram estado de Depressão mínimo e 2 pacientes $(12,5 \%)$ estado de Depressão leve (Quadro 2 e Gráfico 1).

\section{3) Questionário de Qualidade de Vida SF-36}

A avaliação da Qualidade de vida foi feita por meio do instrumento SF-36 que considera 0 o pior valor e 100 o melhor valor esperado na pontuação da avaliação.

O resultado desta avaliação pode ser observado no quadro 3 . A análise destes dados permite concluir que da primeira para a segunda avaliação:

1) Capacidade funcional: $45 \%$ (9) melhoraram; $40 \%$ (8) pioraram; $15 \%$ (3) mantiveram.

2) Limitação por aspectos físicos: $30 \%$ (6) melhoraram; $55 \%$ (11) pioraram; $15 \%$ (3) mantiveram.

\begin{tabular}{|c|c|c|c|}
\hline \multicolumn{4}{|c|}{$\begin{array}{c}\text { Quadro 2. Estados de Depressão dos pacientes } \\
\text { uveal: pré e pós-cirúrgico }\end{array}$} \\
\hline Pacientes & Pré-cirúrgico & $\begin{array}{l}\text { Pós-cirúrgico } \\
3 \text { meses }\end{array}$ & $\begin{array}{l}\text { Pós-cirúrgico } \\
1 \text { ano }\end{array}$ \\
\hline 1 & 10 & 21 & * \\
\hline 2 & 12 & 15 & 7 \\
\hline 3 & 10 & 13 & 15 \\
\hline 4 & 9 & 18 & 8 \\
\hline 5 & 24 & 40 & 7 \\
\hline 6 & 7 & 30 & 5 \\
\hline 7 & 12 & 14 & ** \\
\hline 8 & 5 & 8 & 2 \\
\hline 9 & 9 & 4 & 4 \\
\hline 10 & 14 & 26 & 7 \\
\hline 11 & 5 & 9 & $* * *$ \\
\hline 12 & 13 & 21 & 8 \\
\hline 13 & 13 & 13 & 7 \\
\hline 14 & 28 & 31 & 12 \\
\hline 15 & 12 & 14 & 2 \\
\hline 16 & 8 & 15 & 2 \\
\hline 17 & 5 & 12 & 5 \\
\hline 18 & 11 & 13 & 7 \\
\hline 19 & 13 & 41 & $* * * *$ \\
\hline 20 & 5 & 10 & 5 \\
\hline
\end{tabular}

3) Dor: 70\% (14) melhoraram; 30\% (6) pioraram.

4) Estado geral de saúde: 65\% (13) melhoraram; 35\% (7) pioraram.

5) Vitalidade: 30\% (6) melhoraram; 55\% (11) pioraram; $15 \%$ (3) mantiveram.

6) Aspectos sociais: $30 \%$ (6) melhoraram; $10 \%$ (2) mantiveram; 60\% (12) pioraram.

7) Limitação por aspectos emocionais: 5\% (1) melhoraram; $85 \%$ (17) pioraram e $10 \%$ (2) mantiveram. Este foi o item em que o maior número de pacientes apresentou piora (85\%).

8) Saúde mental: 35\% (7) melhoraram; 5\% (1) mantiveram; $60 \%$ (12) pioraram.

Considerando as três avaliações: $1^{\mathrm{a}}, 2^{\mathrm{a}}, 3^{\mathrm{a}}$ avaliação (16 pacientes):

1) Capacidade funcional: $68,7 \%$ (11) melhoraram; $12,5 \%$ (2) mantiveram; e $18,7 \%$ (3) pioraram.

2) Limitação por aspectos físicos: $68,7 \%$ (11) melhoraram; $6,2 \%$ (1) mantiveram; $25 \%$ (4) pioraram.

3) Dor: $75 \%$ (12) melhoraram; $25 \%$ (4) pioraram.

4) Estado geral de saúde: $62,5 \%$ (10) melhoraram; 37,5\% (6) pioraram.

5) Vitalidade: 68,7\% (11) melhoraram; 31,2\% (5) pioraram.

6) Aspectos sociais: $87,5 \%$ (14) melhorarame $12,5 \%$ (2) pioraram.

7) Limitação por aspectos emocionais: $81 \%$ (13) melhoraram; $6,2 \%$ (1) mantiveram; $12,5 \%$ (2) pioraram.

8) Saúde mental: 87,5\% (14) melhoraram; $12,5 \%$ (2) pioraram.

\section{DISCUSSÃO}

Este estudo teve em sua amostra o predomínio do sexo masculino $(65 \%)$, o que está de acordo com a literatura ${ }^{(7)}$. Prevaleceu, quanto ao grau de escolaridade, o ensino fundamental incompleto $(60 \%)$, o que reflete a faixa social que procura o atendimento médico em hospital universitário. A idade média foi de 52 anos, e $30 \%$ dos pacientes tinham menos de 40 anos quando do diagnóstico. Idade média abaixo de 55 anos tem sido referida em estudos no Brasil ${ }^{(7-8)}$ como o estudo de Hatanaka (1995), em que $40 \%$ dos pacientes tinham menos de 40 anos. O

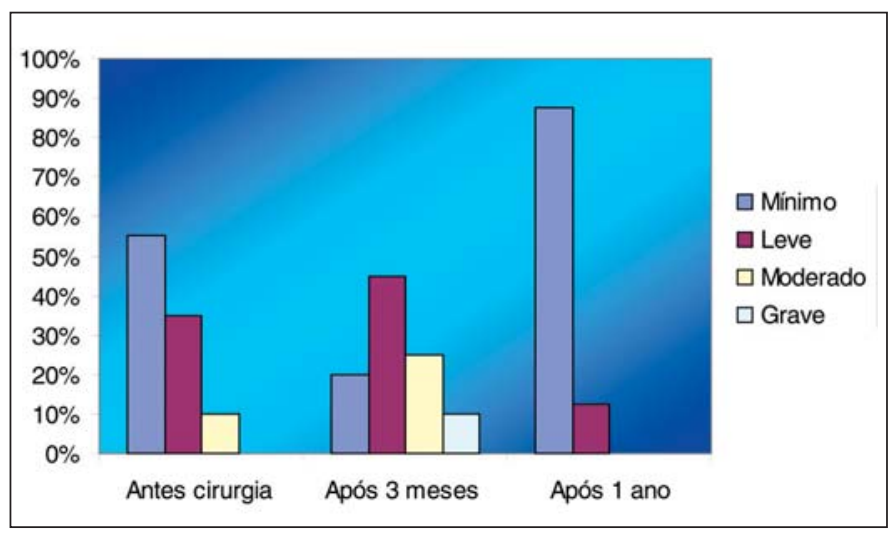

Gráfico 1 - Estados de Depressão dos pacientes com melanoma uveal: pré e pós-cirúrgico 


\begin{tabular}{|c|c|c|c|c|c|c|c|c|c|c|c|c|}
\hline \multicolumn{13}{|c|}{ Quadro 3. SF 36 - Avaliação da Qualidade de Vida nos momentos pré e pós-cirúrgico (continua) } \\
\hline \multirow{2}{*}{$\begin{array}{l}\text { Dados } \\
\text { Pacientes }\end{array}$} & \multicolumn{3}{|c|}{ Capacidade funcional } & \multicolumn{3}{|c|}{ Limitação por aspectos físicos } & \multicolumn{3}{|c|}{ Dor } & \multicolumn{3}{|c|}{ Estado geral de saúde } \\
\hline & $\mathbf{A}$ & B & C & A & B & C & A & B & $\mathbf{C}$ & A & B & C \\
\hline 1 & 75 & 85 & * & 25 & 50 & * & 40 & 60 & * & 40 & 45 & * \\
\hline 2 & 75 & 70 & 40 & 100 & 0 & 25 & 52 & 100 & 100 & 67 & 42 & 82 \\
\hline 3 & 70 & 65 & 65 & 100 & 0 & 0 & 72 & 100 & 74 & 60 & 75 & 72 \\
\hline 4 & 75 & 95 & 100 & 100 & 100 & 100 & 74 & 100 & 100 & 72 & 52 & 67 \\
\hline 5 & 95 & 95 & 95 & 50 & 50 & 75 & 100 & 100 & 100 & 77 & 45 & 72 \\
\hline 6 & 60 & 65 & 65 & 25 & 50 & 100 & 41 & 10 & 100 & 52 & 60 & 82 \\
\hline 7 & 50 & 30 & ** & 0 & 0 & ** & 52 & 31 & ** & 60 & 50 & ** \\
\hline 8 & 90 & 80 & 90 & 50 & 100 & 100 & 84 & 61 & 41 & 42 & 82 & 57 \\
\hline 9 & 85 & 85 & 85 & 25 & 100 & 100 & 62 & 100 & 100 & 82 & 62 & 97 \\
\hline 10 & 80 & 40 & 65 & 0 & 0 & 100 & 41 & 22 & 52 & 25 & 50 & 82 \\
\hline 11 & 80 & 85 & 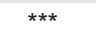 & 50 & 50 & $\star * \star *$ & 62 & 100 & $\star * *$ & 47 & 50 & $\star \star * *$ \\
\hline 12 & 33 & 33 & 55 & 0 & 0 & 50 & 52 & 100 & 64 & 12 & 42 & 77 \\
\hline 13 & 40 & 75 & 50 & 0 & 0 & 0 & 41 & 100 & 41 & 52 & 37 & 77 \\
\hline 14 & 40 & 45 & 65 & 0 & 0 & 50 & 0 & 41 & 41 & 62 & 67 & 37 \\
\hline 15 & 85 & 65 & 90 & 0 & 0 & 75 & 100 & 80 & 100 & 27 & 57 & 65 \\
\hline 16 & 65 & 70 & 75 & 0 & 25 & 100 & 32 & 100 & 100 & 32 & 90 & 87 \\
\hline 17 & 100 & 85 & 100 & 100 & 0 & 100 & 84 & 61 & 100 & 62 & 75,5 & 77 \\
\hline 18 & 80 & 70 & 75 & 50 & 50 & 50 & 41 & 100 & 100 & 65,5 & 82 & 82 \\
\hline 19 & 95 & 85 & 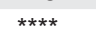 & 25 & 0 & 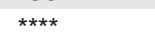 & 52 & 64 & $* \star \star *$ & 47 & 27 & $\star \star \star \star *$ \\
\hline 20 & 50 & 55 & 70 & 100 & 25 & 25 & 100 & 100 & 100 & 65,5 & 77 & 82 \\
\hline
\end{tabular}

National Cancer Institute (USA) em 2004(9) estima a média de idade de pacientes com melanoma uveal entre 55 e 60 anos.

Durante o período de acompanhamento dos pacientes deste estudo, ocorreu uma morte por metástase após um ano e meio da cirurgia de enucleação (sobrevida de 95\%); ocorreram, também, metástases sistêmicas após dois anos e meio da cirurgia em 1 paciente (ainda em acompanhamento) e, um acidente vascular cerebral isolado em 1 paciente, aparentemente sem relação com o câncer. O estudo de Rouberoul et al., na França, relata sobrevida de cinco anos em $82 \%$ dos casos e, em 10 anos, de $72 \%{ }^{(10)}$. O estudo Justin em Cuba, faz referência à sobrevida em 10 anos, igual a $61,78 \%{ }^{(11)}$. Nenhum estudo correlaciona a idade de aparecimento do tumor à sobrevida ou à idade média.

Para compreender fundamentalmente as repercussões emocionais dos pacientes diante do diagnóstico de câncer e indicação cirúrgica, partimos da noção psicanalítica de que a emoção acompanha o ser humano desde o seu primeiro contato com o meio e o influencia ao longo da vida. As emoções interagem com todo e qualquer fenômeno do desenvolvimento humano promovendo a responsabilidade do homem pela própria história. Evidencia-se, portanto, a relevância de se olhar a doença para além da noção de uma etiologia específica e a de integrá-la à compreensão dos aspectos psicológicos, culturais, sociais, entre outros. Psique e soma não podem ser vistos de forma separa$\mathrm{da}^{(12)}$, as emoções precisam ser mobilizadas para que os estados afetivos não sejam camuflados. Muitas vezes os dramas ocultos se desenrolam dentro de um clima indefinido e angustiante.

A preocupação com o diagnóstico de uma forma de câncer pouco difundida entre os leigos e que leva a uma mutilação que pode modificar a aparência física da face, motivou este estudo que procurou medir o estado emocional e a qualidade de vida como repercussões de uma proposta terapêutica. Além do mais, a preocupação com a reconstrução da aparência facial foi muito valorizada nesse grupo de pacientes que participou concomitantemente do estudo de reconstrução da cavidade anoftálmica. Foram todos assistidos pelo mesmo cirurgião e dispunham de prótese intracavitária desenhada para permitir boa motilidade do implante e excelência estética.

Para a compreensão da avaliação de Ansiedade antes e após a cirurgia de enucleação por meio do IDATE é importante diferenciar dois conceitos distintos de Ansiedade. O Estado de Ansiedade refere-se ao estado emocional transitório ou à condição do organismo humano, caracterizado por sentimento desagradável de tensão e apreensão, conscientemente percebido. O Traço de Ansiedade refere-se a diferenças individuais relativamente estáveis, uma tendência habitual e, talvez, em parte, geneticamente determinada.

As avaliações da Ansiedade mostraram que em 17 pacientes, o Estado de Ansiedade, esteve mais elevado do que o Traço de Ansiedade. Três meses após a realização da cirurgia, o Estado de Ansiedade aumentou consideravelmente em relação ao Estado anterior; 12 dos 17 pacientes acentuaram mais o Estado de Ansiedade. Este resultado nos conduz à reflexão de que a doença ficou mais presente na vida desses indivíduos, causou insegurança em relação à saúde, medos e conflitos. Pode-se deduzir que o impacto da cirurgia desencadeou uma Ansiedade maior e uma preocupação acentuada diante das expectativas de vida, o que é natural, dada a gravidade da doença, e mostra que os 


\begin{tabular}{|c|c|c|c|c|c|c|c|c|c|c|c|c|}
\hline \multirow{3}{*}{$\begin{array}{l}\text { Dados } \\
\text { Pacientes }\end{array}$} & \multicolumn{12}{|c|}{ Quadro 3. SF 36 - Avaliação da Qualidade de Vida nos momentos pré e pós-cirúrgico (continuação) } \\
\hline & \multicolumn{3}{|c|}{ Vitalidade } & \multicolumn{3}{|c|}{ Aspectos sociais } & \multirow{2}{*}{$\frac{\text { Limitação }}{\text { A }}$} & por aspectos & \multirow{2}{*}{$\frac{s \text { emocionais }}{\mathrm{C}}$} & \multicolumn{3}{|c|}{ Saúde mental } \\
\hline & A & B & C & A & B & C & & B & & A & B & C \\
\hline 1 & 60 & 60 & * & 75 & 50 & * & 0 & 0 & * & 60 & 52 & * \\
\hline 2 & 80 & 70 & 70 & 100 & 87,5 & 87,5 & 0 & 0 & 33,3 & 56 & 48 & 72 \\
\hline 3 & 80 & 70 & 20 & 62,5 & 37,5 & 12,5 & 66,6 & 0 & 0 & 64 & 48 & 36 \\
\hline 4 & 80 & 35 & 80 & 75 & 100 & 100 & 66,6 & 33,3 & 100 & 76 & 40 & 80 \\
\hline 5 & 65 & 100 & 65 & 25 & 25 & 80 & 0 & 0 & 66,6 & 40 & 48 & 72 \\
\hline 6 & 60 & 60 & 80 & 75 & 37,5 & 100 & 33,3 & 0 & 100 & 48 & 40 & 80 \\
\hline 7 & 80 & 35 & ** & 100 & 62,5 & ** & 100 & 0 & ** & 92 & 53,3 & ** \\
\hline 8 & 95 & 70 & 75 & 100 & 100 & 100 & 100 & 0 & 100 & 92 & 72 & 84 \\
\hline 9 & 70 & 70 & 85 & 62,5 & 50 & 100 & 66,6 & 66,6 & 100 & 76 & 80 & 96 \\
\hline 10 & 45 & 10 & 75 & 62,5 & 12,5 & 100 & 0 & 0 & 66 & 16 & 16 & 60 \\
\hline 11 & 80 & 70 & 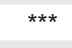 & 50 & 75 & 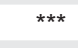 & 33,3 & 0 & $\star * \star *$ & 68 & 60 & $* \star \star *$ \\
\hline 12 & 45 & 60 & 60 & 37,5 & 37,5 & 62,5 & 0 & 0 & 66,6 & 48 & 60 & 68 \\
\hline 13 & 50 & 60 & 65 & 0 & 0 & 37,5 & 0 & 66,6 & 66,6 & 44 & 56 & 72 \\
\hline 14 & 0 & 45 & 55 & 12,5 & 37,5 & 62,5 & 0 & 0 & 66,6 & 0 & 48 & 60 \\
\hline 15 & 55 & 60 & 75 & 100 & 75 & 100 & 66,6 & 66,6 & 66,6 & 40 & 64 & 76 \\
\hline 16 & 65 & 40 & 80 & 100 & 37 & 100 & 66,6 & 33,3 & 100 & 64 & 32 & 92 \\
\hline 17 & 50 & 75 & 90 & 87,5 & 100 & 100 & 66,6 & 0 & 33,3 & 88 & 72 & 88 \\
\hline 18 & 65 & 70 & 80 & 37,5 & 62,5 & 100 & 0 & 0 & 66,6 & 52 & 48 & 72 \\
\hline 19 & 55 & 0 & $\star \star * * *$ & 100 & 0 & $* \star * * *$ & 33,3 & 0 & $* * * *$ & 64 & 24 & 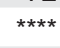 \\
\hline 20 & 80 & 90 & 75 & 100 & 87,5 & 100 & 100 & 66,6 & 100 & 96 & 100 & 88 \\
\hline
\end{tabular}

pacientes estavam sintonizados com o que estava acontecendo com eles, não negavam nem racionalizavam.

Um ano após a realização da cirurgia, o Traço de Ansiedade foi reavaliado, a fim de se observar se a maneira habitual do indivíduo de lidar com a vida mudou nesse período. Constatamos que, em oito dos pacientes avaliados, o Traço de Ansiedade diminuiu, o que permite considerar que essas pessoas passaram a lidar de forma mais amena com os problemas do cotidiano em comparação com a forma com que lidavam com eles antes do diagnóstico e da cirurgia.

Quanto à depressão, os resultados mostraram antes da cirurgia que 11 dos 20 pacientes estavam no estado mínimo de Depressão ou não apresentavam depressão; sete apresentaram Depressão leve e apenas dois tiveram Depressão moderada. Três meses após a cirurgia, houve aumento do estado de Depressão em 18 pacientes: nove tiveram Depressão leve; cinco, Depressão moderada: quatro, estado mínimo de Depressão e dois, Depressão grave. Quadros depressivos ${ }^{(13)}$ podem ser desencadeados ante a presença de doença grave. Acreditamos que aos três meses da cirurgia a situação da doença tenha ficado mais presente. A depressão em pacientes com câncer pode aparecer no início, diante do diagnóstico, ou após a cirurgia e outros tratamentos. Após um ano de cirurgia, 14 dos 16 pacientes avaliados apresentaram um estado mínimo de depressão.

A preocupação com a qualidade de vida das pessoas ocupa um espaço maior entre profissionais da saúde, que, em geral, têm em seus objetivos de trabalho a preocupação de ouvir e sentir o paciente, bem como de elaborar um programa de orientação.

$\mathrm{Na}$ avaliação da Qualidade de Vida, os pacientes revelaram antes da cirurgia prejuízos no domínio da limitação por aspectos emocionais, seguido pela limitação por aspectos físicos. Já após três meses da cirurgia, os pacientes demonstraram prejuízos também em outros domínios, como vitalidade, aspectos sociais e saúde mental e, com maior destaque, limitação por aspectos emocionais. Os prejuízos mais acentuados na segunda avaliação indicam que a dificuldade de retomar a vida tornouse mais presente. As limitações foram mais percebidas. Assim, os aspectos físicos, vitalidade, aspectos sociais e saúde mental foram afetados, em especial e com destaque os emocionais. Este dado assinala que o abalo com a cirurgia ocasionou traumas emocionais que repercutiram de forma extremamente considerável. O estudo sobre reações Psicológicas e Qualidade de Vida com melanoma uveal ${ }^{(14)}$, constatou o aumento da Ansiedade e do estresse em pacientes cirúrgicos. O SF-36 aliado a um questionário para doenças oculares foi utilizado em estudos para avaliação de pacientes com melanoma uveal e observaram boa correlação entre os questionários, sendo possível detectar os aspectos em conflito nos pacientes avaliados.

Um ano após a cirurgia, evidenciou-se uma recuperação nos aspectos do SF-36 na maioria dos pacientes. Neste estudo, os dados obtidos no SF-36 revelaram toda a dificuldade que os pacientes enfrentam com a doença e com a cirurgia. Autores que avaliaram o impacto da doença e seu tratamento (enucleação, remoção do olho, e terapia de radiação sem a remoção do olho) na Qualidade de vida por meio do SF-36 $6^{(15-16)}$ concluíram que a enucleação ou terapia de radiação pode ter diferentes efeitos psicológicos e fisiológicos em cada paciente que a recebe, e que vão refletir em sua qualidade de vida. 


\section{CONCLUSÃO}

O presente estudo revelou-nos que o pior momento psicológico no processo de tratamento de um paciente pela remoção do bulbo ocular ocorreu três meses após o ato cirúrgico. A nosso ver, fica a questão: será que o cirurgião valoriza da mesma forma esse momento? É importante dizer que, no início, o paciente vivência o medo e, com o passar do tempo, as experiências vão modificando as vivências, e a pessoa vai digerindo melhor, em pequenas doses, o acontecido. Assim, acompanhar o paciente no sentido de ver e estar presente em todos os momentos em que ele retorna para o atendimento, pode contribuir e ajudá-lo em seu processo de reabilitação. Fixamos, portanto, a importância de nos atermos às respostas dos pacientes, à forma como eles nos comunicam sua realidade. No momento em que muitas mudanças estão em jogo na vida dessas pessoas, o que soa com maior intensidade é o resgate da vida, do continuar. Aos profissionais da saúde, compete o compromisso de proporcionar formas de atendimento que visem à informação, à orientação e ao suporte emocional prestado aos pacientes, por meio da efetiva prática da medicina.

\section{ABSTRACT}

Purpose: To evaluate prospectively the emotional repercussions in the individual with uveal melanoma and surgical indication in three phases: diagnosis and preoperative, postsurgery and late postsurgery. Methods: Clinical qualitative study based on the following instruments: STAI - State, Trait Anxiety Inventory; BDI - Beck Depression Inventory; Quality of Life Questionaire SF-36. Results: The sample of this study consisted of 20 patients, 13 males and seven females, with average age of 52 years. Before surgery: the patients appear fragile and impacted by diagnosis and treatment. The state of depression indicates a minimum to light state. Anxiety has the state increased in relationship to Trait. Quality of Life has the emotional and physical aspects affected. Three months after surgery: the Depression shows to light to serious state. Anxiety has the state considerably increased. Quality of Life has most aspects affected: the physical, vitality, social, mental health and emotional aspects being the most affected. One year after surgery: the State of Anxiety decreases considerable. The Trait of Anxiety is maintained in most patients when compared to the first evaluation. Depression shows a minimum state. Quality of Life has most of its aspects balanced. Conclusions: After three months of surgery the patients appear more fragile with adaptation difficulty, Anxiety and Depression increasing. This period revealed to be the worst moment. One year after surgery, they appear more structured and with a more balanced Quality of Life.

Keywords: Uveal neoplasy/complications; Uveal neoplasms/ psychology; Ocular enucleation/etiology; Ocular enucelation/psychology; Anxiety/psychology; Depression/psychology; Quality of life

\section{REFERÊNCIAS}

1. Kornfeld DS. Consultation-liaison psychiatry: contributions to medical practice. Am J Psychiatry. 2002;159(12):1964-72. Comment in: Am J Psychiatry. 2002; 159(12):1961-3.

2. Turato ER. Tratado da metodologia da pesquisa clínico-qualitativa: construção teórico-epistemológica, discussão comparada e aplicação nas áreas da saúde e humanas. Petrópolis: Vozes; 2003. 685p.

3. Biaggio AMB, Natalício L. Manual para Inventário de Ansiedade Traço-Estado (IDATE). Rio de Janeiro: Centro Editor de Psicologia Aplicada (CEPA); 1979.

4. Cunha JA. Manual da versão em português das Escalas Beck. São Paulo: Casa do Psicólogo; 2001.

5. Ciconelli RM, Ferraz MB, Santos W, Meinão I, Quaresma MR. Tradução para a língua portuguesa e validação do questionário genérico de avaliação de Qualidade de vida SF-36 (Brasil SF-36). Rev Bras Reumatol. 1999;39(3):143-50.

6. Caumo W, Schmidt AP, Schneider CN, Bergmann J, Iwamoto CW, Bandeira D, et al. Risk factors for preoperative anxiety in adults. Acta Anaesthesiol Scand. 2001;45(3):298-307.

7. Oliveira BV, Ramos GHA, Abib FC. Neoplasias malignas do bulbo ocular e demais conteúdos orbitários no Hospital Erasto Gaertner - (1973/1986). An Oftalmol. 1989;8(1):122-8

8. Hatanaka M, Freitas MAFC, Nishimoto IN, Erwenne CM. Melanoma de coróide: estudo de distribuição segundo faixa etária. São Paulo: Fundação Antônio Prudente; 1995.

9. American Cancer Society. Cancer reference Information. What is eye cancer? [texto on the Internet]. Atlanta: American Cancer Society; 2004. [cited 2004 Sep 14]. Available from: http://www.cancer.org/docroot/CRI/content/ CRI_2_4_1x_What_is_eye_cancer_74.asp

10. Rouberol F, Roy P, Kodjikian L, Gérard JP, Jean-Louis B, Grange JD. Survival, anatomic, and functional long-term results in choroidal and ciliary body melanoma after ruthenium brachytherapy (15 years' experience with betarays). Am J Ophthalmol. 2003;137(5):893-900.

11. Melgares Ramos M de los A, Santos SD, Puig Mora M, Cruz Mojarrieta J, Zárate ME, Cordies Justín N. Melanoma de coróides, estúdio de la casuística en el INOR en el período de 1980-1996. Rev Cuba Oncol. 1998;14(3):149-54.

12. McDougall J. Teatros do corpo: o psicossoma em psicanálise. Trad. PHB Rondon. 2a ed. São Paulo: Martins Fontes; 1996.

13. Mattos P. Depressão e doença clínica. In: Doris HM, Bernik M, Mattos P, Cordás TA. Recuperação em depressão. São Paulo: Livre; 2003. p.63-71.

14. Brandberg Y, Kock E, Oskar K, af Trampe E, Seregard S. Psychological reactions and quality of life in patients with posterior uveal melanoma treated with ruthenium plaque therapy or enucleation: a one year follow-up study. Eye. 2000;14(Pt 6):839-46.

15. Meliá BM, Moy CS, McCaffrey L. Quality of life in patients with choroidal Melanoma: a pilot study. Ophthalmic Epidemiol. 1999;6(1):19-28.

16. Moy CS, Meliá MB. The COMS quality of life assessment in the collaborative ocular melanoma study: design and methods. COMS-QOLS Report No. 1. COMS Quality of Life Study Group. Ophthalmic Epidemiol. 1999;6(1):5-17. 